\title{
RUN-TO-RUN OPTIMIZATION OF BATCH EMULSION POLYMERIZATION
}

\author{
G. François, B. Srinivasan and D. Bonvin \\ Institut d'Automatique, \\ École Polytechnique Fédérale de Lausanne, \\ CH-1015 Lausanne, Switzerland.
}

\begin{abstract}
Run-to-run optimization exploits the repetitive nature of batch processes to adapt the optimal operating policy in the presence of uncertainty. For problems where terminal constraints play a dominant role in optimization, the system can be operated close to the optimum simply by satisfying terminal constraints. The optimal input is parameterized by using the knowledge of the shape of the optimal solution, and the input parameters are adapted in the presence of uncertainty to meet the terminal constraints. When the number of input parameters is greater than the number of terminal constraints, an adaptation methodology based on a projection matrix derived from a gain matrix between the input parameters and the terminal constraints is proposed. The run-to-run optimization scheme is illustrated in simulation for the minimization of the batch time of an emulsion polymerization process with terminal constraints on conversion and number average molecular weight.
\end{abstract}

Keywords: Batch reactor, Emulsion polymerization, Dynamic optimization, Run-to-run optimization, Batch-to-batch optimization.

\section{INTRODUCTION}

Emulsion polymerization probably represents the most subtle of all polymerization processes. Despite its relative complexity, batch emulsion polymerization is the method of choice whenever specifications on conversion, particle size or molecular weight distribution are stringent. In fact, virtually $100 \%$ conversion can be achieved with this polymerization process (Kiparissides, 1996).

Dynamic optimization is a useful tool to reduce production costs and guarantee satisfaction of tight specifications. Optimization of batch homogeneous polymerizations has been widely studied in the literature. Typical optimization problems include minimization of batch time (Butala et al., 1992) and minimization of molecular weight distribution (Scali et al., 1995). However, the op- timization of batch emulsion polymerization has not been studied extensively since standard optimization tools are model-based and the class of processes considered is rather difficult to model. The available studies often involve implementing a profile that has been determined off-line on the basis of a model (Gentric et al., 1999). However, this approach may not lead to optimality when there is uncertainty regarding the initial conditions and the model parameters, or in the presence of disturbances. Industry typically copes with uncertainty by introducing a fair amount of conservatism in order to guarantee constraint satisfaction.

Since batch-end measurements are readily available, a run-to-run approach based on these measurements can help reduce this conservatism by exploiting the repetitive nature of batch processes. The measurements can be used in an iterative 
manner to find the optimal operating conditions despite uncertainty, especially when constraints play a dominant role in the optimal profile. Among the various run-to-run optimization techniques, an approach that does not use a process model at the implementation level is preferred. In particular, the invariant-based optimization technique that is based on tracking appropriate references to guarantee optimality is well suited for this application (Srinivasan et al., 2001b).

The case study presented in this paper concerns the optimization of the reactor temperature profile for the emulsion copolymerization of styrene $/ \alpha$-methylstyrene in a batch reactor. The reactor temperature is controlled by means of a cooling fluid circulating through a jacket. The operational constraints include bounds on the reactor and jacket temperatures. The performance is ensured via lower bounds on both the final conversion and the final number average molecular weight. The optimization objective is then to minimize the batch time necessary to meet the performance specifications by manipulating the reactor temperature. A run-to-run optimization strategy will be implemented in the presence of uncertainty (parametric uncertainty in the propagation rate constant and in the transfer to monomer rate constant).

The paper is organized as follows. Section 2 proposes an approach for selecting the directions in the input parameter space that drive the system to the terminal constraints optimally. Moving in these directions ensures maximal cost improvement with respect to meeting the terminal constraints. Section 3 presents a simplified dynamic model of the polymerization reactor. The shape of the optimal profile and the resulting input parameterization are discussed in Section 4. Run-to-run optimization results are presented in Section 5, while Section 6 concludes the paper.

\section{OPTIMIZATION WITH UNCERTAINTY AND OFF-LINE MEASUREMENTS}

In this section, invariant-based optimization, a method for adapting the optimal solution under uncertainty using measurements, will be reviewed (Bonvin et al., 2001; Srinivasan et al., 2001b). It will be assumed that only off-line (batchend) measurements are available. So, the procedure presented below is tuned for the run-to-run adaptation using batch-end measurements as in (Srinivasan et al., 2001a).

Consider the following optimization problem:

$$
\begin{aligned}
& \min _{u(t)} \phi\left(x\left(\theta, t_{f}\right)\right) \\
& \text { s.t. } \dot{x}=F(x, \theta, u), \quad x(0)=x_{o}
\end{aligned}
$$

$$
T\left(x\left(\theta, t_{f}\right)\right) \leq 0
$$

where $x$ are the states ( $n$-dimensional), $u$ the input ( $m$-dimensional), $F$ the system equations, $x_{o}$ the initial conditions, $\theta$ the set of uncertain parameters, $\phi$ the terminal cost function, and $T$ the terminal constraints ( $\tau$-dimensional). Without loss of generality, assume that all the terminal constraints are active.

The optimal input of (1) is a concatenation of various arcs. The input in each arc can be parameterized as a function of the states and/or additional parameters. The input parameterization $\pi$ also includes the switching time between different arcs. Then, the input can be written as $u=\mathcal{U}(\pi, x)$, where $\pi$ is $n_{\pi}$-dimensional. Note that $n_{\pi} \geq \tau$ so as to be able to meet all the terminal constraints. The necessary conditions of optimality are given by (Srinivasan et al., 2001b):

$$
T=0, \quad \frac{\partial \phi}{\partial \pi}+\nu^{T} \frac{\partial T}{\partial \pi}=0
$$

where $\nu$ ( $\tau$-dimensional $)$ are the Lagrange multipliers for the constraints. The necessary conditions consist of two parts: (i) the constraint part and (ii) the sensitivity part.

The basic idea of the invariant-based optimization approach is to enforce the conditions of optimality in the presence of uncertainty by adjusting the values of $\pi$ on the basis of process measurements. Adaptation of $\pi$ to satisfy the constraints $T=0$ is straigtforward. However, meeting the sensitivity conditions is less obvious due to (i) absence of direct measurement of the sensitivities, and (ii) the presence of the unknown Lagrange multiplier $\nu$.

Yet, the fact that comes handy is that there is usually considerably more to gain by satisfying the constraints than by reducing the sensitivities to zero. So, the approach taken here is to simply adapt $\pi$ so as to meet the constraints. The question that arises is: "which combinations of $\pi$ need to be adjusted to push the system to the terminal constraints?" A method based on the gain matrix relating the input parameters and the terminal constraints is investigated for the same.

The method proposed in this paper divides the directions in the input parameter space into those that do not affect the constraints and those that do. The division is based on the gain matrix, $G: \pi \rightarrow T$, between the input parameters $\pi$ and the constraints $T, G=\frac{\partial T}{\partial \pi}$, which is of dimension $\tau \times n_{\pi}$. Let $\operatorname{rank}(G)=\bar{\tau} \leq \tau$. In most cases, the constraints are independent and $\bar{\tau}=\tau$.

Singular value decomposition gives $G=U S V^{T}$ where $U$ has dimension $\tau \times \tau, S$ has dimension $\tau \times n_{\pi}$ and $V$ has dimension $n_{\pi} \times n_{\pi}$. The matrices $U, S$, and $V$ can be partitioned into: 


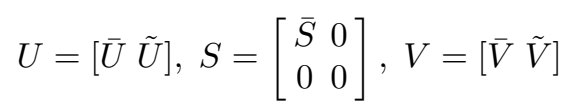

where $\bar{U}$ and $\bar{V}$ correspond to the first $\bar{\tau}$ columns of the respective matrices and $\tilde{U}$ and $\tilde{V}$ to the remaining columns. $\bar{S}_{G}$ is the $\bar{\tau} \times \bar{\tau}$ submatrix of $S$. Due to the structure of $S, G=\bar{U} \bar{S} \bar{V}^{T}$. $\tilde{V}$ is of dimension $n_{\pi} \times\left(n_{\pi}-\bar{\tau}\right)$ and corresponds to the directions that do not affect the constraints. So, by defining $\bar{\pi}=\bar{V}^{T} \pi$ and $\tilde{\pi}=\tilde{V}^{T} \pi$, there results $\frac{\partial T}{\partial \bar{\pi}}=\bar{U} \bar{S}$ and $\frac{\partial T}{\partial \tilde{\pi}}=0 . \bar{\pi}$ is of dimension $\bar{\tau}$ and $\tilde{\pi}$ of dimension $\left(n_{\pi}-\bar{\tau}\right)$.

The elements of $\bar{\pi}$ will be adapted using batch-end measurements. The pseudo-inverse of the $\bar{\tau} \times \tau$ matrix $\frac{\partial T}{\partial \bar{\pi}}$ can be used for decoupling and then decentralized integral controllers can be used. $\tilde{\pi}$ is kept constant at its conservative off-line determined value. The adaptation law is given by:

$$
\begin{aligned}
& \bar{\pi}(k)=\bar{\pi}(k-1)+\bar{S}^{-1} \bar{U}^{T} K T(k) \\
& \pi(k)=\bar{V} \bar{\pi}(k)+\tilde{V} \tilde{\pi}
\end{aligned}
$$

where $K$ is a diagonal gain matrix of dimension $\tau \times \tau$. The two above equations can be put together in a compact form as shown below:

$$
\pi(k)=\pi(k-1)+P G^{+} K T(k)
$$

where the pseudo-inverse of $G$ is given by, $G^{+}=$ $\bar{V} \bar{S}^{-1} \bar{U}^{T}$ and $P$ is a projection matrix, $P=\bar{V} \bar{V}^{T}$. Note that the singular value decomposition is used to determine the projection matrix $P$, i.e, the directions that are adapted to satisfy the constraints. The block diagram representation of the scheme is shown in Figure 1.

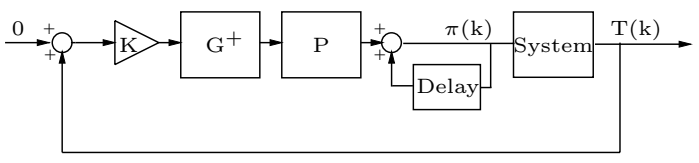

Figure 1. Block Diagram of the Adaptation Scheme

The reasons for using a decomposition based on the gain matrix $G$ are twofold:

(1) The Lagrange variables $\nu$ can be isolated in necessary conditions. In the space of $\bar{\pi}$ and $\tilde{\pi}$, the necessary conditions read:

$T=0, \quad \frac{\partial \phi}{\partial \tilde{\pi}}=0, \quad$ and $\quad \frac{\partial \phi}{\partial \bar{\pi}}+\nu^{T} \frac{\partial T}{\partial \bar{\pi}}=0$

The parameters $\bar{\pi}$ and $\tilde{\pi}$ are adjusted to meet the conditions $T=0$ and $\frac{\partial \phi}{\partial \tilde{\pi}}=0$, respectively. The last condition is only used to determine the Lagrange multipliers $\nu$ and, thus, need not be considered.

(2) The direction $\bar{\pi}$ corresponds to the direction of maximum reduction in cost that can be achieved when the constraints are moved by an infinitesimally small amount and the parameters are only adjusted to satisfy the constraints. In other words, it is equivalent to the steepest descent direction in the presence of constraints.

\section{TENDENCY MODEL FOR THE POLYMERIZATION PROCESS}

A detailed model of the reaction is very difficult to obtain due to the presence of several phases in the reactor. Even if such a model can be obtained, it consists of a large number of differential equations and parameters that are difficult to estimate. The most appropriate model for optimization is a tendency model that describes the main phenomena involved.

\subsection{Polymerization mechanism}

The mechanism of emulsion polymerization has been classically divided into three intervals (Smith and Ewart, 1948) :

Interval 1 Particle nucleation: Free radicals are produced in the aqueous phase by initiator decomposition. They are captured by the micelles, where the polymerization begins. This interval ends with the disappearance of the micelles.

Interval 2 Particle growth with monomer saturation: Particles grow as the monomer diffuses from the monomer droplets towards the particles at a rate faster than monomer consumption. Thus, the particles are saturated with monomer. This interval ends with the disappearance of monomer droplets.

Interval 3 Particle growth without monomer sup$p l y$ : The reaction proceeds and the monomer concentration in the particles decreases.

The following assuptions are made in building this model (Gentric et al., 1999) :

- Styrene and $\alpha$-methylstyrene are both hydrophobic monomers : nucleation is supposed to be heterogeneous and only micellar nucleation is considered.

- Propagation, transfer to monomer, termination reactions in the aqueous phase and radical desorption are neglected.

- There is no more than one radical per polymer particle, as termination in particles is very rapid compared to radical entry

- Emulsifier molecules are adsorbed in monomolecular layers at the polymer particles surface.

- Gel effect does not take place .

\subsection{Tendency model}

Initiator decomposition dynamics are neglected and the initiator concentration is supposed to be 
constant : $I=I_{0}$. Quasi-steady-state approximation leads to the following tendency model :

$$
\begin{array}{lll}
\dot{M}=-R_{p}=-k_{p} M_{p} \frac{N_{p}}{N_{a}} \bar{n}, & M(0) & =M_{0} \\
\dot{N}_{p}=\frac{R_{i} N_{a}}{1+\left(\frac{\epsilon N_{p}}{S N_{a}}\right)}, & N_{p}(0)=1 \\
\dot{Q}_{0}=\frac{R_{i} \bar{n} N_{p}}{N_{p}+\left(\frac{S}{\epsilon}\right)}+k_{t r M} M_{p} \frac{N_{p}}{N_{a}}, & Q_{0}(0)=0
\end{array}
$$

where $M$ is the overall monomer concentration, (styrene and $10 \%$ in mass of $\alpha$-methylstyrene), $M_{p}$ is the concentration of the monomer in the particles, $N_{p}$ is the number of particles, $Q_{0}$ is the zeroth order moment of the molecular weight distribution, $N_{a}$ is the Avogadro number and $\bar{n}$ is the average number of radicals per particle. $R_{i}$ represents the initiator decomposition rate, $R_{i}=2 f_{i} k_{d} I_{0}$, where $f_{i}$ is the efficiency factor of initiator decomposition. The expression of $M_{p}$ is different in various intervals:

$$
M_{p}= \begin{cases}M_{p_{c}} & \text { if } X \leq X_{c} \\ \frac{(1-X) \rho_{m}}{\left[(1-X)+X \rho_{m} / \rho_{p}\right] M_{M}} & \text { if } X>X_{c}\end{cases}
$$

where $X(t)=1-\frac{M(t)}{M(0)}$ is the global conversion, and $X_{c}$ the critical conversion. The difference in the expression of $M_{p}$ is due to the fact that, at the end of Interval 2, there are no droplets left and so during Interval $3, M_{p}$ decreases with conversion. The concentration of the emulsifier is $S=S_{0}-k_{v}\left(X M_{0}\right)^{\frac{2}{3}} N_{p}^{\frac{1}{3}}$ where $S_{0}$ is the initial emulsifier concentration, $M_{0}$ the initial monomer concentration, and $k_{v}$ a constant.

Number average molecular weight $M_{n}(t)$ can be defined as $M_{n}(t)=M_{M} \frac{M(0)-M(t)}{Q_{0}(t)}$ and the kinetic coefficients $k_{p}, k_{d}$ and $k_{t r M}$ follow the Arrhenius's law: $k_{p}=k_{p_{0}} e^{-\frac{E_{p}}{R T}}, \quad k_{d}=k_{d_{0}} e^{-\frac{E_{d}}{R T}}, \quad k_{t r M}=$ $k_{t r M_{0}} e^{-\frac{E_{t r M}}{R T}}$.

The dynamics of the heating jacket are given by:

$\dot{T}=-\frac{V \Delta H}{m_{r} C_{p}} R_{p}+\frac{U A\left(T_{j}-T\right)}{m_{r} C_{p}}, \quad T(0)=T_{0}$

$\dot{T}_{j}=\frac{F_{j}\left(T_{j i n}-T_{j}\right)}{V_{j}}-\frac{U A\left(T_{j}-T\right)}{\rho_{j} V_{j} C_{j}}, T_{j}(0)=T_{j_{0}}$

where $T_{j}$ is the jacket temperature, and $T_{j i n}$ is the jacket inlet temperature. The parameters of the model are given in Table 1.

\section{CHARACTERIZATION OF THE OPTIMAL SOLUTION}

Since the reactor temperature influences the system directly and efficiently, it represents a natural choice for the decision variable. On the one hand, increasing the reactor temperature accelerates the reaction but, on the other hand, it
Table 1. Model parameters

\begin{tabular}{cc|cc}
\hline$f_{i}$ & 0.5 & $\bar{n}$ & 0.5 \\
$\epsilon$ & $10^{-16}$ & $N_{a}$ & $6.0210^{23}$ \\
$k_{v}$ & $10^{-7} \mathrm{~g} /(\mathrm{mol} . \mathrm{l})^{\frac{2}{3}}$ & $E_{d}$ & $140.2 \mathrm{~kJ} / \mathrm{mol}$ \\
$k_{d_{0}}$ & $4.510^{6} \mathrm{~s}^{-1}$ & $E_{p}$ & $29 \mathrm{~kJ} / \mathrm{mol}$ \\
$k_{p_{0}}$ & $5.710^{6} \mathrm{l} /(\mathrm{mol} . \mathrm{s})$ & $E_{t r M}$ & $85 \mathrm{~kJ} / \mathrm{mol}$ \\
$k_{t r M_{0}}$ & $1.510^{11} \mathrm{l} /(\mathrm{mol} . \mathrm{s})$ & $m_{r} C_{p}$ & $4.151 \mathrm{~kJ} / \mathrm{K}$ \\
$U A$ & $6.4 \mathrm{~J} /(\mathrm{K.s})$ & $F_{j} / V_{j}$ & $0.817 \mathrm{~s}-1$ \\
$V \Delta H$ & $-66.9 \mathrm{kJ.l} / \mathrm{mol}$ & $\rho_{j} V_{j} C_{j}$ & $1.946 \mathrm{~J} / \mathrm{K}$ \\
$\rho_{m}$ & $0.91 \mathrm{~kg} / l$ & $\rho_{p}$ & $1.1 \mathrm{~kg} / \mathrm{l}$ \\
$M_{M}$ & $105.41 \mathrm{~g} / \mathrm{mol}$ & $M_{p_{c}}$ & $5.38 \mathrm{~mol} / l$ \\
$X_{c}$ & 0.422 & $M_{0}$ & $2.16 \mathrm{~mol} / l$ \\
$I_{0}$ & $3.710^{-3} \mathrm{~mol} / l$ & $S_{0}$ & $4.432 \mathrm{~g} / \mathrm{l}$ \\
$T_{0}$ & $343 \mathrm{~K}$ & $T_{j_{0}}$ & $343 \mathrm{~K}$ \\
\hline
\end{tabular}

modifies the structure and the properties of the polymer, thus leading to shorter polymer chains and lower molecular weights. Since the batch time is free, it represents another decision variable.

The reactor temperature is considered as the manipulated variable, and it is supposed that a controller is designed such that it allows ideal reactor temperature control. Also, the optimization problem will be formulated such that the jacket dynamics (10) are implicitly expressed as a constraint on the rate of heat removal.

\subsection{Problem formulation}

The optimization problem consits of minimizing the batch time subject to bounds on the reactor temperature, bounds on the jacket inlet temperature, terminal constraints on conversion and number average molecular weight. The problem can be mathematically expressed as follows:

$$
\min _{T(t), t_{f}} J=t_{f}
$$

subject to dynamic equations (8)

$$
\begin{aligned}
& T_{\min } \leq T(t) \leq T_{\max } \\
& T_{j i_{\min }} \leq T_{j i n}(t) \\
& X\left(t_{f}\right) \geq X_{f_{d}} \\
& M_{n}\left(t_{f}\right) \geq M_{n f_{d}}
\end{aligned}
$$

where $T_{\min }$ and $T_{\max }$ represents the bounds on the reactor temperature, $T_{j i n_{\text {min }}}$ a lower bound on the jacket inlet temperature, $X_{f_{d}}$ and $M_{n f_{d}}$ minimal desired values at final time for conversion and number average molecular weight, respectively. $T_{j i n}(t)$, which is adjusted by the reactor temperature controller, can be computed from (10) and thus $T_{j i n}(t)=f n\left(M, N_{p}, T, \dot{T}, \ddot{T}\right)$.

\subsection{Sequence of arcs and input parameterization}

In invariant-based optimization, the choice of the references to be tracked is based on a characterization of the optimal solution. If the type and sequence of arcs that constitute the optimal solution and the set of active terminal constraints do not vary with the uncertainty, feedback controllers 
can be used to push the system closer to the active constraints and regulate certain sensitivities around zero (Srinivasan et al., 2001b).

The nature of the optimal solution for the emulsion copolymerization of styrene/ $\alpha$-methylstyrene in a batch reactor can be determined by analysis of the tendency model. The aforementioned compromise between conversion and molecular weight has an immediate consequence: the optimal solution will not necessarily be on input bounds or path constraints, and some $\operatorname{arc}(\mathrm{s})$ will exist to represent this intrinsic compromise. The solution can be constructed qualitatively and it consists in the following four arcs :

Arc 1 : During Interval 1, which is the nucleation step, it is assumed that polymer particles do not grow. Thus, it is possible to accelerate the nucleation by increasing temperature without decreasing molecular weight. As a result, the reactor temperature is set at its maximum value $T_{\max }$. Arc 1 is thus the constraint-seeking arc $T(t)=T_{\max }$.

Arc 2 : As soon as particles start to grow, the optimal reactor temperature is somewhere between $T_{\min }$ and $T_{\max }$ due to the intrinsic compromise. The second arc implements the transition between Arc 1 and the following arcs in minimum time. The best way to jump from $T_{\max }$ to some intermediate temperature is to decrease $T$ as fast as possible, which is limited by the lower bound on $T_{j i n}$. Arc 2 is also a constraint-seeking arc with $T_{j i n}(t)=$ $f n\left(M, N_{p}, T, \dot{T}, \ddot{T}\right)=T_{j i n_{\text {min }}}$.

Arc 3 : During Interval 2, the rate of reaction is nearly constant. Any increase in temperature will favour conversion, but the average molecular weight will decrease, and vice versa. The solution is thus to keep the temperature constant at some intermediate optimal value that can be determined using tools from differential geometry (this is not presented here). Arc 3 is thus a compromise-seeking arc.

Arc 4 : During Interval 3, the rate of reaction decreases as a function of $M_{p}$. The optimal solution therefore consists of increasing the temperature to compensate for the decrease in reaction rate. Thus, Arc 4 is also a compromise-seeking arc.

In addition, the two terminal constraints regarding conversion and molecular weight must be active for the batch to be optimal.

A way to determine the various arcs is to use the conditions of optimality from Pontryagin's Maximum Principle together with tools from differential geometry such as Lie brackets. For each arc, an analytical expression was found that confirmed the qualitative analysis given above. Especially, it was shown that $\dot{T}=0$ during Arc 3 . It was noticed that Arc 4 is an unstable arc (the temperature derivative is a function of $T^{2}$ ). However, for the class of desired conversions, only the quasi-linear part of this arc is used.

Since an analytical expression for each arc is available, the optimal solution can be parameterized using only the switching times between these arcs, the free final time and the initial temperature of the fourth arc (the latter cannot be determined using analytical tools). Furthermore, a sensitivity analysis shows that the last arc has little influence (less than $0.3 \%$ ) on the cost and thus can be discarded. This way, the optimal temperature profile has only three arcs and can be parameterized using the two switching times $t_{s w_{1}}$ and $t_{s w_{2}}$ and the final time $t_{f}$, as shown in Figure 2. These three parameters can be adjusted on a run-to-run basis using batch-end measurements in order to satisfy the two active terminal constraints on conversion and number average molecular weight.

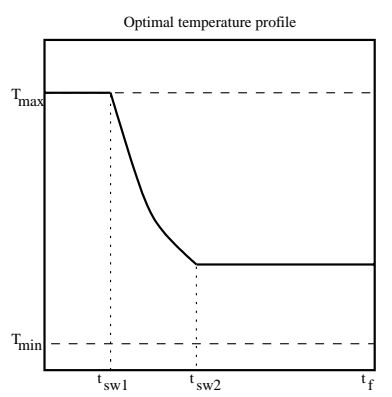

Figure 2. Nominal optimal input without Arc 4

\section{OPTIMIZATION RESULTS}

The approach proposed in Section 2 for choosing the update directions to meet the two terminal constraints is investigated in simulation. Assuming $\pm 15 \%$ variation in $k_{p_{0}}$ and $\pm 10 \%$ variation in $k_{t r M_{0}}$, a conservative strategy that will serve as a reference for the run-to-run schemes is first obtained.

The presence of $5 \%$ zero-mean gaussian measurement noise is considered. As a result, a backoff (conservatism) is introduced in order not to violate the terminal constraints. $X_{f_{d}}^{*}$ and $M_{n f_{d}}^{*}$ represent the conservative set points provided to the controller. The numerical values of the constraints and controller set points are given in Table 2 . With the backoffs used, the controller gains are tuned such that no constraint violation occurs.

Three uncertainty cases are described in Table 3 and the corresponding results are presented in Table 4. In Case (a), the uncertainty is relatively large and the improvement through adaptation is nearly 40\%. In Cases (b) and (c), the uncertainty is lower and, although the improvement is smaller, it can still be quite significant. 


\begin{tabular}{cc|cc} 
Constraint & Value & Constraint & Value \\
\hline$T_{\min }$ & $313 \mathrm{~K}$ & $T_{\max }$ & $343 \mathrm{~K}$ \\
$T_{j \text { in }}$ & $293 \mathrm{~K}$ & & \\
$X_{f_{d}}$ & $60 \%$ & $M_{n f_{d}}$ & $2 \times 10^{6} \mathrm{~g} / \mathrm{mol}$ \\
$X_{f_{d}}^{*}$ & $63 \%$ & $M_{n f_{d}}^{*}$ & $2.1 \times 10^{6} \mathrm{~g} / \mathrm{mol}$ \\
\hline
\end{tabular}

Table 2. Values of constraints and controller set points

\begin{tabular}{ccc} 
Case & $k_{p_{0}}$ & $k_{t r M_{0}}$ \\
\hline (a) & $+15 \%$ & $-10 \%$ \\
(b) & $+5 \%$ & $-5 \%$ \\
(c) & $-5 \%$ & $+5 \%$
\end{tabular}

Table 3. Values of uncertain parameters

Though the run-to-run optimization scheme takes about 20 runs to converge to the optimal values, the major part of the optimization is done in the first few batches. The run-to-run evolution of the cost function and the constraints are shown in Figures 3 and 4.

\begin{tabular}{ccc} 
Case & $5^{\text {th }}$ batch & $30^{\text {th }}$ batch \\
\hline (a) & $1.31(29.8)$ & $1.14(38.9)$ \\
(b) & $1.50(19.5)$ & $1.34(28.1)$ \\
(c) & $1.67(10.0)$ & $1.59(14.5)$ \\
\hline
\end{tabular}

Table 4. Final time in hours (improvement in \% from the conservative solution).

A remark regarding the speed of convergence: although the matrix $G$ changes with the operating point, the proposed adaptation uses a fixed $G$. So, if $G$ is recomputed a few times during the adaptation process, the speed of convergence can be improved.

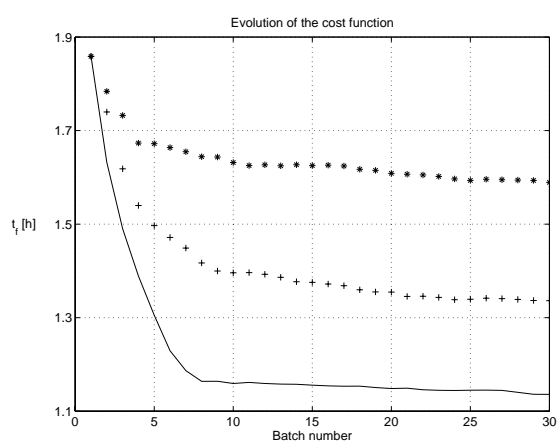

Figure 3. Evolution of the cost function, Case (a)

$$
(-) \text {, Case }(b)(+) \text {, Case }(c)(*)
$$

\section{CONCLUSION}

This work has demonstrated the effectiveness of run-to-run optimization for the batch emulsion copolymeriztion of styrene $/ \alpha$-methylstyrene. A scheme has been proposed for choosing the update directions in input parameter space to effectively meet the terminal constraints. This way, batch performance can be nearly maximized when the cost is dominated by terminal constraints. The approach is based on the gains between the input parameters and the terminal constraints, the gains

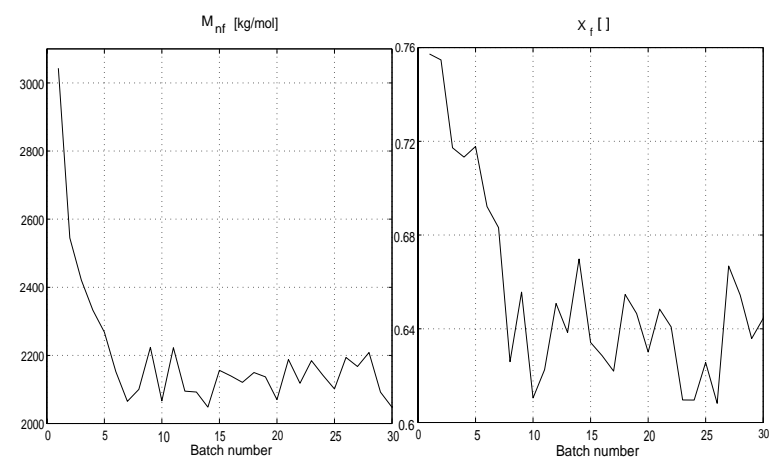

Figure 4. Evolution of the average number molecular weight and of the conversion : Case (a)

being calculated around the conservative input profile. Two possible extensions can be envisioned: i) the gains are recomputed a few times during the adaptation process in order to speed up convergence, and ii) information regarding the effect that uncertainty has on the values of the optimal input parameters can be used to find uncertaintyrelated update directions.

\section{REFERENCES}

Bonvin, D., B. Srinivasan and D. Ruppen (2001). Dynamic optimization in the batch chemical industry. In: Chemical Process Control - 6 . Tucson, AZ.

Butala, D., W.R. Liang and K.Y. Choi (1992). Multiobjective dynamic optimization of a batch free radical polymerization process catalyzed by mixed initiator systems. J. Appl. Polym. Science 44, 1759-78.

Gentric, C., F. Pla, M. A. Latifi and J. P. Corriou (1999). Optimization and nonlinear control of a batch emulsion polymerization. Chem. Eng. Journal 75(1), 31-46.

Kiparissides, C. A. (1996). Polymerization reactor modeling : A review of recent developments and future directions. Chem. Eng. Science 51(10), 1637-59.

Scali, C., R. Ciari, T. Bello and G. Maschio (1995). Optimal temperature for the control of the product quality in batch polymerization : Simulation and experimental results. J. Appl. Polym. Science 39, 945-959.

Smith, W.V. and R.H. Ewart (1948). Kinetics of emulsion polymerization. J. Chem. Phys. 16, $592-599$.

Srinivasan, B., C. J Primus, D. Bonvin and N. L. Ricker (2001a). Run-to-run optimization via generalized constraint control. Control Eng. Practice 9, 911-919.

Srinivasan, B., D. Bonvin, E. Visser and S. Palanki (2001b). Dynamic optimization of batch processes: II. Handling uncertainty using measurements. Comp. Chem. Eng. p. Submitted. 